OPEN ACCESS

Edited by:

Brian G. Drew,

Baker IDI Heart and Diabetes Institute,

Australia

Reviewed by:

Thomas E. Adrian,

United Arab Emirates University,

United Arab Emirates

Laura Maria Frago,

Autonomous University of Madrid,

Spain

*Correspondence:

Marcia J. Abbott

mabbott@chapman.edu

Specialty section:

This article was submitted to

Integrative Physiology,

a section of the journal

Frontiers in Physiology

Received: 30 June 2016 Accepted: 30 November 2016 Published: 20 December 2016

Citation:

Krolopp JE, Thornton SM and Abbott MJ (2016) IL-15 Activates the Jak3/STAT3 Signaling Pathway to Mediate Glucose Uptake in Skeletal Muscle Cells. Front. Physiol. 7:626

doi: 10.3389/fphys.2016.00626

\section{IL-15 Activates the Jak3/STAT3 Signaling Pathway to Mediate Glucose Uptake in Skeletal Muscle Cells}

\author{
James E. Krolopp ${ }^{1}$, Shantaé M. Thornton ${ }^{1}$ and Marcia J. Abbott ${ }^{1,2 *}$ \\ ${ }^{1}$ Department of Health Sciences and Kinesiology, Crean College of Health and Behavioral Sciences, Chapman University, \\ Orange, CA, USA, ${ }^{2}$ Department of Biological Sciences, Human and Evolutionary Biology Section, Dana and David Dornsife \\ College of Letters, Arts and Sciences, University of Southern California, Los Angeles, CA, USA
}

Myokines are specialized cytokines that are secreted from skeletal muscle (SKM) in response to metabolic stimuli, such as exercise. Interleukin-15 (IL-15) is a myokine with potential to reduce obesity and increase lean mass through induction of metabolic processes. It has been previously shown that IL-15 acts to increase glucose uptake in SKM cells. However, the downstream signals orchestrating the link between IL-15 signaling and glucose uptake have not been fully explored. Here we employed the mouse SKM C2C12 cell line to examine potential downstream targets of IL-15-induced alterations in glucose uptake. Following differentiation, $\mathrm{C} 2 \mathrm{C} 12$ cells were treated overnight with $100 \mathrm{ng} / \mathrm{ml}$ of $\mathrm{IL}-15$. Activation of factors associated with glucose metabolism (Akt and AMPK) and known downstream targets of IL-15 (Jak1, Jak3, STAT3, and STAT5) were assessed with IL-15 stimulation. IL-15 stimulated glucose uptake and GLUT4 translocation to the plasma membrane. IL-15 treatment had no effect on phospho-Akt, phospho-Akt substrates, phospho-AMPK, phospho-Jak1, or phospho-STAT5. However, with IL-15, phospho-Jak3 and phospho-STAT3 levels were increased along with increased interaction of Jak3 and STAT3. Additionally, IL-15 induced a translocation of phospho-STAT3 from the cytoplasm to the nucleus. We have evidence that a mediator of glucose uptake, HIF1 $\alpha$, expression was dependent on IL-15 induced STAT3 activation. Finally, upon inhibition of STAT3 the positive effects of IL-15 on glucose uptake and GLUT4 translocation were abolished. Taken together, we provide evidence for a novel signaling pathway for IL-15 acting through Jak3/STAT3 to regulate glucose metabolism.

Keywords: myokines, skeletal muscle glucose uptake, Jak/STAT, AMPK, IL-15

\section{INTRODUCTION}

Obesity is a major problem in our modern society, its prevalence continues to grow, and it is linked to many disease processes, such as diabetes, cardiovascular disease, and certain cancers (Félix-Redondo et al., 2013; Ogden et al., 2014). A multitude of studies have been underway to uncover regulators of metabolism with the intent of treating and/or preventing obesity and its associated disease states (Hampton, 2012; Pedersen and Febbraio, 2012; Egan and Zierath, 2013; Febbraio, 2014). It has long been known that increasing skeletal muscle (SKM) mass and activity 
are routes to induce energy expenditure for the reduction of adiposity and improvements in insulin resistance (Ivy et al., 1986; Kraegen et al., 1989; Pedersen and Febbraio, 2012). Recently, SKM has proven to possess functions beyond muscle contraction (Pedersen and Febbraio, 2012). Many cytokines secreted from skeletal muscle, termed "myokines," have been identified and muscle contraction appears to be a major stimulator of their release (Pedersen, 2013; Raschke and Eckel, 2013; Catoire et al., 2014; Eckardt et al., 2014). Multiple myokines, BDNF5, FGF21, irisin, and interleukin-6 (IL-6), among others, have been shown to exert their positive metabolic actions in an endocrine/paracrine manner (Fisher ffolliott et al., 2012; Raschke and Eckel, 2013; Catoire et al., 2014; Indrakusuma et al., 2015).

Interleukin-15 (IL-15) is a myokine that has shown promise for the prevention and/or treatment of obesity and metabolic disorders (Alvarez et al., 2002; Argilés et al., 2009; Quinn and Anderson, 2011; Sun and Liu, 2015). Historically, IL-15 has been regarded as an activator of natural killer (NK) cells and has been viewed as pro- and anti-inflammatory with antitumorigenic potential (Castillo and Schluns, 2012; Lutz and Quinn, 2012). IL-15 belongs to a 4- $\alpha$-helix bundle cytokine family alongside a variety of other interleukins that are within this family (Budagian et al., 2006; Waldmann, 2015). Expression of the gene encoding for IL-15 results in the translation of two isoforms, a short 21-amino acid and a long 48-amino acid signal peptide (Quinn and Anderson, 2011). The long IL-15 isoform combines intracellularly with its receptor, IL-15R $\alpha$, prior to secretion from SKM (Budagian et al., 2006; Quinn and Anderson, 2011). Subsequently, the cytokine-receptor complex either transpresents or binds to the $\beta$ and $\gamma$ chains of the IL- 2 receptor on its target tissues for initiation of IL-15 signaling (Budagian et al., 2006; Castillo and Schluns, 2012). Alternatively, IL-15 can form a heterodimer with the IL-2 receptor independent of IL-15R $\alpha$ (Castillo and Schluns, 2012). Importantly, there is evidence that the appearance of IL-15 in circulation increases following exercise, in humans and rodents, although this notion is somewhat controversial (Gray and Kamolrat, 2011; Catoire et al., 2014; Rinnov et al., 2014; Crane et al., 2015; Pierce et al., 2015).

Increased circulating levels of IL-15 have been implicated in stimulating expression of mitochondrial associated factors, such as PPARs and SIRT1, in mouse SKM (Almendro et al., 2008; Quinn et al., 2012, 2013; O'Connell and Pistilli, 2015). Additionally, IL-15 has the ability to stimulate both glucose uptake and fatty acid oxidation in SKM cells (Busquets et al., 2006). Further, transgenic mice with increased IL-15 in circulation display an increased endurance capacity phenotype (Quinn et al., 2012). However, recent in vivo studies have questioned the relevance of IL-15 secretion following exercise in humans (Pierce et al., 2015). Although it has been demonstrated that IL-15 induces metabolic pathways in SKM, the discrete molecular mediators of these effects have not been fully defined. The most well studied pathway for IL-15 action is the janus kinase activation of signal transducer and activator of transcription proteins (Jak/STAT) signaling pathway (Waldmann, 2015; Ye, 2015). Upon IL-15 binding to the IL-2 receptor, Jak isoforms (Jak1 and/or Jak3) are auto-phosphorylated and in turn induce phosphorylation of STAT3 and/or STAT5 (Ye, 2015). Overall the Jak/STAT signaling pathway has a large number of intracellular functions with the potential to effect energy metabolism in many cell types (Frias and Montessuit, 2013; Richard and Stephens, 2014; Ye, 2015). Alternatively, pathways aside from the Jak/STAT signaling cascade have been linked to IL-15 action (Stone et al., 2011; Zhao and Huang, 2012; Crane et al., 2015; Waldmann, 2015). For instance, it has been established that the PI3K/Akt pathway becomes activated downstream of IL15 action (Budagian et al., 2006; Zhao and Huang, 2012; Lai et al., 2013; Waldmann, 2015; Ye, 2015). Additionally, a link between the energy sensing enzyme AMP-activated protein kinase (AMPK) and IL-15 has been established by us and others (Abbott et al., 2012; Turcotte and Abbott, 2012; Crane et al., 2015). Both Akt and AMPK signaling exert beneficial effects on substrate metabolism such as glucose uptake and fatty acid oxidation in SKM cells, in line with IL-15 action (Thorell et al., 1999). However, little is known regarding the signaling pathway downstream of IL-15-IL-2 receptor interaction, to mediate substrate metabolism in SKM cells.

Overall, there is strong evidence that IL-15 plays a positive role in mediating SKM substrate utilization (Busquets et al., 2006; Argilés et al., 2009; Quinn and Anderson, 2011). However, the signaling molecules responsible for orchestrating IL-15 action on energy metabolism have yet to be firmly established in SKM. The purpose of this study was to identify the molecular pathways that mediate the downstream effects of IL-15 signaling in SKM cells. Here, we demonstrate that IL-15 increases glucose uptake and GLUT4 translocation, through induction of the Jak3/STAT3 signaling pathway in SKM cells.

\section{METHODS}

\section{C2C12 Cell Culture}

The immortalized mouse SKM fibroblast line, C2C12 (Sigma), was cultured in DMEM supplemented with $10 \%$ fetal bovine serum (FBS; Sigma), 1\% Penicillin-Streptomycin (10,000 U/mL; Corning), and $0.1 \%$ Amphotericin B (Corning). At $80 \%$ confluence, cells were induced toward differentiation to mature myotubes with DMEM supplemented with $2 \%$ horse serum (Sigma) and $1 \mu \mathrm{M}$ insulin (Sigma) for 6 days. Differentiation was confirmed by visualization of myotube formation. On the fifth day of differentiation cells were treated with $100 \mathrm{ng} / \mathrm{ml}$ of recombinant IL-15 (Genscript) for $24 \mathrm{~h}$, as previously described (Abbott and Turcotte, 2014; Thornton et al., 2016).

\section{Glucose Uptake Assay}

Glucose uptake was measured in fully differentiated $\mathrm{C} 2 \mathrm{C} 12$ cells using a non-radioactive fluorometric assay, as previously described (Leira et al., 2002; Zou et al., 2005; Kanwal et al., 2012). Briefly, following differentiation in 6 well plates, cells were serum starved in growth media for $2 \mathrm{~h}$ and cells were treated with either vehicle control, IL-15 $100 \mathrm{ng} / \mathrm{ml}$, insulin (100 nM), a STAT3 inhibitor (100 $\mu \mathrm{M}$; S31-201; Sigma), or IL-15 + S3120 with or without $50 \mu \mathrm{M}$ 2-(N-(7-Nitrobenz-2-oxa-1,3-diazol4-yl)Amino)-2-Deoxyglucose (2-NBDG) (Cayman Chemical) in Krebs-Henseleit buffer containing $0.1 \%$ fatty acid free BSA 
(Sigma) and $2000 \mathrm{mg} / \mathrm{L}$ of glucose at $37^{\circ} \mathrm{C}$ with $5 \% \quad \mathrm{CO}_{2}$ (Siddiquee et al., 2007; Kelly et al., 2010). Following 2 h, the cells were lysed in PBS-Triton-X100 buffer and florescence was measured using a microplate reader with excitation $488 \mathrm{~nm}$ and emission $520 \mathrm{~nm}$. The concentration of NBDG uptake was calculated from a standard curve of NBDG in lysis buffer. Values were normalized to the protein content present in the cell lysates.

\section{Western Blot Analysis}

C2C12 cells were lysed in a modified RIPA buffer supplemented with protease inhibitors (Pierce) (Abbott et al., 2009). For nuclear fractionation, cells were lysed with a commercially available kit and cytosolic and nuclear fractions were recovered (Pierce) (Abbott et al., 2012). Total protein was assessed using a standard Bradford assay (BioRad). Approximately $20 \mu \mathrm{g}$ of protein from the cell homogenate preparations were separated on a $4-12 \%$ gradient gel (GenScript) via SDS-PAGE. Proteins were transferred onto Immobilon-P polyvinylidene difluoride (PVDF) membranes and blocked with 5\% BSA in Tween-TBS for $1 \mathrm{~h}$ The membranes were then incubated $\left(4^{\circ} \mathrm{C}\right)$ in $5 \%$ BSA in TweenTBS with antibodies $(1: 1000)$ against phospho-AMPK-Thr172, total AMPK, phospho-Akt-Thr308, phospho-Akt substrate, total Akt, phospho-Jak1, total Jak1, phospho-Jak3, total Jak3, phosphoSTAT3-Tyr705, total STAT3, phospho-STAT5, total STAT5 (Cell Signaling), GLUT4 (Santa Cruz), and GAPDH (Sigma). Following overnight incubation, the membranes were then probed with a secondary antibody (Genscript, 1:2000; or Thermo, $1: 10,000)$. Blots were then washed and subjected to enhanced chemiluminescence (Pierce). Band density was quantified using Image $J$ and normalized to control samples. Membranes were stripped in $0.5 \mathrm{M} \mathrm{NaOH}$ for $15 \mathrm{~min}$ at room temperature, followed by three $5 \mathrm{~min}$ washes in TBS-T, and subsequently probed for total proteins and subsequently GAPDH (Sigma) was used as a loading control.

\section{Immunofluorescence}

After overnight IL-15 experimental treatment, the $\mathrm{C} 2 \mathrm{C} 12$ cells were fixed with ice cold methanol. Cells were blocked with 5\% BSA in PBS-Tween followed by a series of washes. Cells were incubated overnight in a humidified chamber with primary antibodies (GLUT4 or pSTAT3) at $4^{\circ} \mathrm{C}$. Alexaflour488 (Molecular Probes) secondary antibodies were used and DAPI was used as a nuclear stain. Cells were imaged using a Zeiss inverted microscope and images were captured with an Axiovision camera. Quantification of GLUT4 on the plasma membrane and co-localization of STAT3 to the nuclei was performed using Image J and Fiji software (Schindelin et al., 2012; Schneider et al., 2012).

\section{Immunoprecipitation}

After the experimental treatments, cells were lysed as described in Western blot analysis and Jak3 specific antibodies (Cell Signaling) were added to the lysate $(250 \mathrm{ug})$ and incubated overnight at $4^{\circ} \mathrm{C}$, as previously described (Abbott et al., 2009; Bogachus and Turcotte, 2010; Ahmadian et al., 2011). Protein A/G agarose beads were added and the slurry was incubated for 2-4h at $4^{\circ} \mathrm{C}$ (Santa Cruz Biotechnology). The immunoprecipitates were collected by centrifugation and pellets were washed with PBS buffer, and the final supernatants were resuspended in SDS-sample buffer (Genscript). Subsequently western blotting procedures were carried out as previously described and antibodies specific to STAT3 (Cell Signaling) were used. Ponceau $S$ was applied to the membranes to verify equal protein loading.

\section{Real Time PCR}

Standard RNA isolation procedures were performed on the cells following the 6 day treatment protocol, as previously described (Pedersen and Febbraio, 2012; Ogden et al., 2014). Briefly, cells were lysed with Trizol reagent and chloroform was added to separate the RNA from the DNA and protein fractions. RNA was precipitated from the clear phase of the Trizol-chloroform mixture, followed by centrifugation at $12,000 \mathrm{~g}$ at $4^{\circ} \mathrm{C}$, with isopropanol. The RNA pellet was washed with $75 \%$ ethanol, centrifuged at $7500 \mathrm{~g}$ for $5 \mathrm{~min}$, at $4^{\circ} \mathrm{C}$, and the pellets were air-dried. Using RNAse-free water, the pellets were resuspended and the RNA purity and concentration were quantified using a Nano-Drop spectrophotometer. Reverse transcription of RNA to cDNA was performed on 2 ug of RNA using SuperScript reverse transcriptase VILO kit. Real time qPCR was performed on the cDNA, using SYBR-green in a 96 well plate. Primer sequences for HIF1 $\alpha$, SOCS3, and GAPDH are listed in Table 1. GAPDH was used as an internal control and the ddCT method was used to calculate gene expression levels.

\section{Statistics}

Statistical analysis was carried out using GraphPad Prism 6.0 software. Values are displayed as means \pm SEM. Each experiment consisted of 3 internal replicates and then were repeated 2-3 times for a total $n=6-9$ for each experimental group. IL-15 treatment values were compared to vehicle control cells and a Student's $T$-test was calculated with $P<0.05$ considered to be statistically significant. For glucose uptake assessments a One-way ANOVA was used with a Fisher's post hoc analysis and a $P$-value of $<0.05$ was considered to be statistically significant.

\section{RESULTS}

\section{IL-15 Promotes GLUT4 Translocation and Glucose Uptake}

In line with previously reported data (Busquets et al., 2006), IL-15 treatment resulted in an increase in glucose uptake

TABLE 1 | Primer sequences used for qPCR analysis.

\begin{tabular}{ll}
\hline Gene & Sequence \\
\hline$H I F 1 \alpha-F$ & GCACTAGACAAAGTTCACCTGAGA \\
$H I F 1 \alpha-R$ & CGCTATCCACATCAAAGCAA \\
SOCS3-F & ATTCGCTTCGGGACTAGC \\
SOCS3-R & AACTGCTGTGGGTGACCAT \\
Gapdh-F & AGGTCGGTGTGAACGGATTG \\
Gapdh-R & TGTAGACCATGTAGTGAGGTCA
\end{tabular}




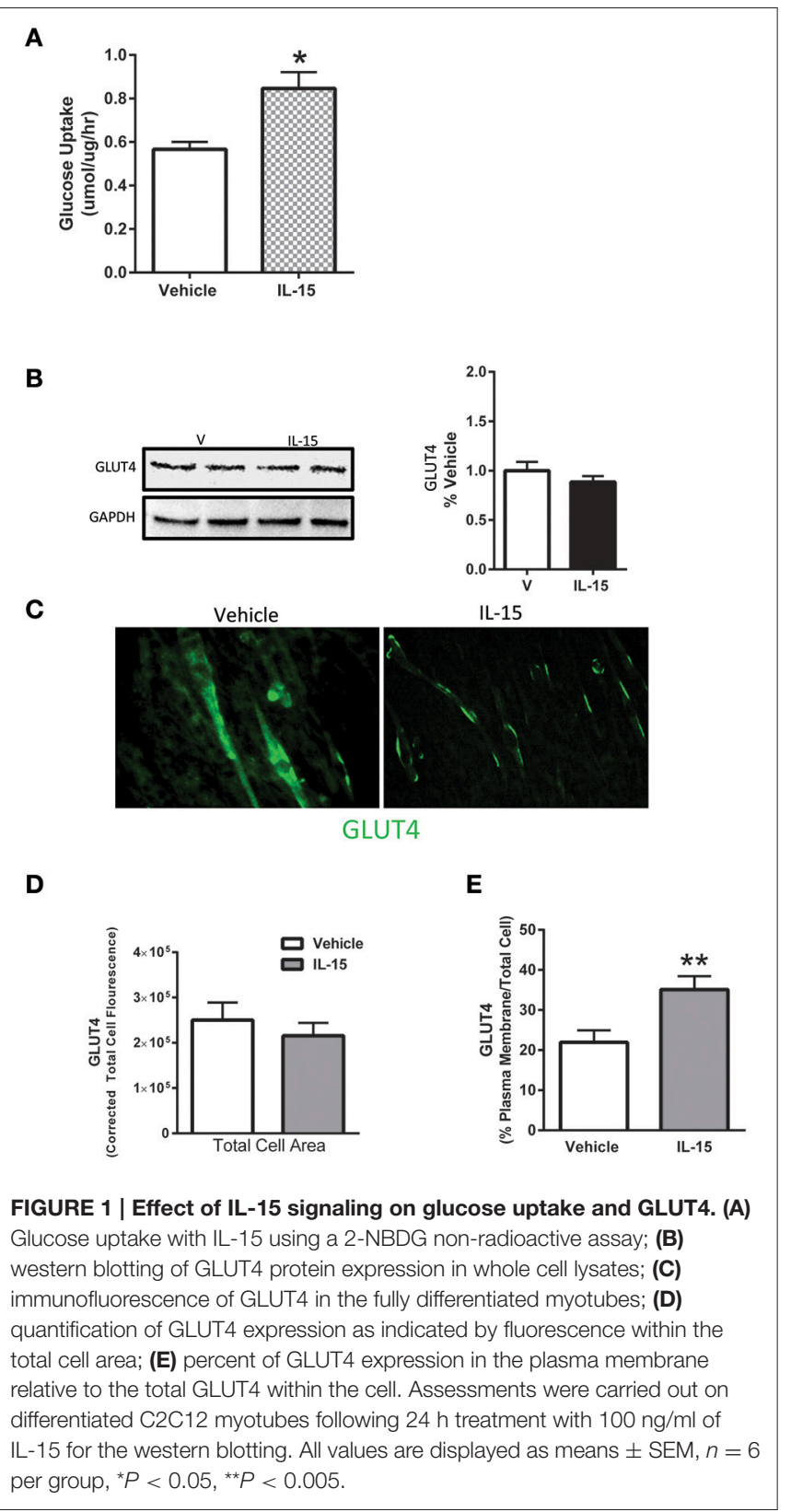

by $49 \%$ when compared to the control SKM cells $(P<$ 0.05; Figure 1A). However, unlike mRNA expression data reported by others (Busquets et al., 2006), there was no significant effect of IL-15 on the total cell protein content of GLUT4 $(P>0.05$; Figure 1B). Conversely, IL-15 treatment stimulated a translocation of GLUT4 from the intracellular compartment to the plasma membrane of the fully differentiated SKM myotubes (Figure 1C). Similar to the western blot expression there were no differences in the quantifiable immunofluorescence of GLUT4 in total cell area $(P>0.05$; Figure 1D). However, the percentage of GLUT4 present on the plasma membrane relative to the total GLUT4 expression was significantly greater in the IL-15 treated cells $(P<0.05$; Figure 1E).

\section{IL-15 Mediated Effects on Enzyme Activation and Protein Expression of IL-2R Targets}

Despite previous evidence that IL-15 may signal through Akt in lymphocytes, we were unable to detect any alterations in phospho-Akt or total Akt protein expression $(P>0.05$; Figure 2A, (Lai et al., 2013; Waldmann, 2015)). Further, the phosphorylation state of downstream substrates (TBC1D1 and TBC1D4) of Akt were also not altered with IL-15 $(P>$ 0.05; Figure 2A). AMPK is a master metabolic regulator and is confirmed to stimulate GLUT4 translocation along with glucose uptake (Winder and Hardie, 1999; Abbott et al., 2009). Additionally, a link between AMPK and IL-15 has been identified by us and others (Abbott et al., 2012; Crane et al., 2015). However, there were no alterations in phosphorylated AMPK at Thr179 or total protein expression of AMPK with IL-15 treatment $(P>0.05$; Figure 2B).

Because IL-15 shares a receptor with IL-2, we attempted to examine potential signaling molecules directly downstream of the IL-2 receptor in SKM cells (Budagian et al., 2006; Waldmann, 2015). A widely studied route of IL-15 signaling is through activation of the Jak/STAT pathway (Mishra et al., 2014b; Waldmann, 2015). However, IL-15 treatment failed to induce an increase in phosphorylation of Jak1 at Tyr1022/1023 $(P>0.05$; Figure 2C). Likewise, there were no effects of IL-15 treatment in regards to total protein expression of Jak1 (Figure 2C). Conversely, in the presence of IL-15, phosphorylation of Jak3 at Tyr980/981 was increased (130\%) with no alterations in its total protein expression $(P<0.05$; Figure 2D).

STAT5 is a known down-stream effector of Jak3 signaling, but phosphorylation of STAT5 at Tyr694 remained unchanged with the IL-15 treatment protocol $(P>0.05$; Figure 3A). A less studied target of Jak3 is STAT3 and we measured increased phosphorylation of STAT3 at Tyr705, nearly 4-fold, with IL$15(P<0.05$; Figure 3B). We also measured increased $(57 \%)$ interaction between Jak3 and STAT3 in the presence of IL-15 $(P<0.05$; Figure 3C). STAT3 has been shown to translocate to the nucleus, upon phosphorylation at Tyr705, to carry out its transcriptional activities (Tammineni et al., 2013). Here we measured a 3-fold increase in phosphorylated (Tyr705) STAT3 in the nuclear fraction as assessed by western blotting and immunofluorescence $(P<0.05$; Figures $4 \mathbf{A}, \mathbf{B})$. The increases in phospho-STAT3 on the nuclear fraction corresponded to a $62 \%$ reduction of phospho-STAT3 in the cytosolic fraction $(P<0.05$; Figure 4A).

\section{Involvement of STAT3 in Mediating IL-15 Induced Glucose Uptake}

In order to determine the necessity of STAT3 activation for mediating IL-15 induced glucose uptake, we used a STAT3 inhibitor. Inhibition of STAT3 in the SKM cells was verified by western blotting of pSTAT3 $(P<0.05$; Figure 5A). When STAT3 was inactivated, the IL-15 induced increases in glucose uptake were abolished when compared to vehicle control cells $(P<0.05$; Figure 5B). It should be noted that addition of the vehicle control (DMSO) in the inhibitor studies yielded 


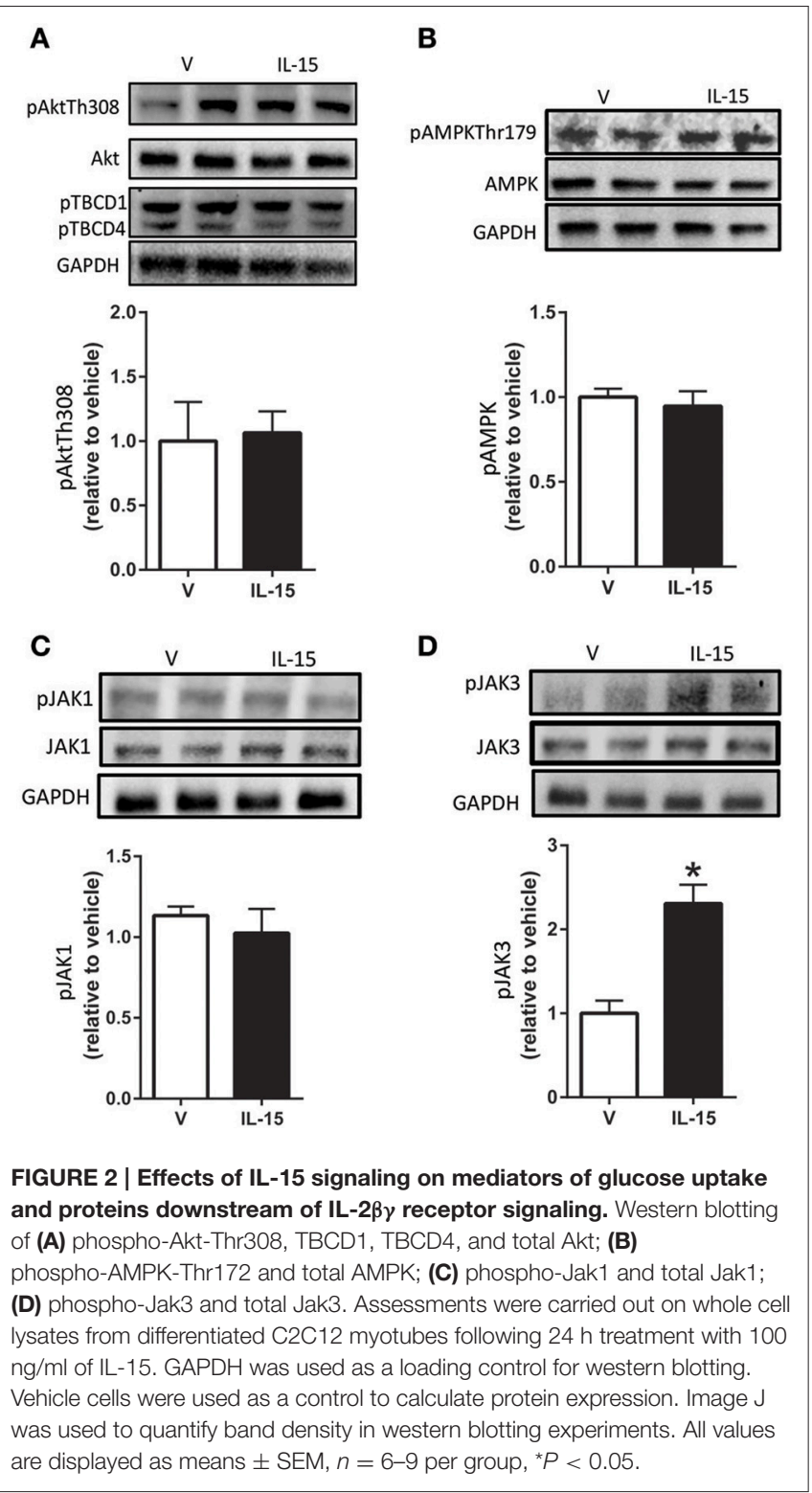

alterations in the relative amounts of glucose uptake when compared to non-DMSO treated cells (Figure 1A). STAT3 has previously been shown to mediate glucose up through HIF1 $\alpha$ induced translocation of GLUT4 (Demaria et al., 2010; Sakagami et al., 2014). Indeed IL-15 induced an increase in mRNA expression levels of HIF $1 \alpha$ and these effects were prevented with STAT3 inhibition in the presence of IL-15 $(P<0.05$; Figure 5C). Conversely, IL-15 had no effect on SOCS3 mRNA expression levels, while as expected, inhibition of STAT3 yielded reductions in SOCS3 expression levels (Figure 5D). Inhibition of STAT3 had no effects on total cellular GLUT4 expression ( $P>0.05$; Figure 6A). However, the IL15 induced translocation of GLUT4 to the plasma membrane was abolished with STAT3 inhibition in combination with IL-15, as measured by percentage of GLUT4 on the plasma
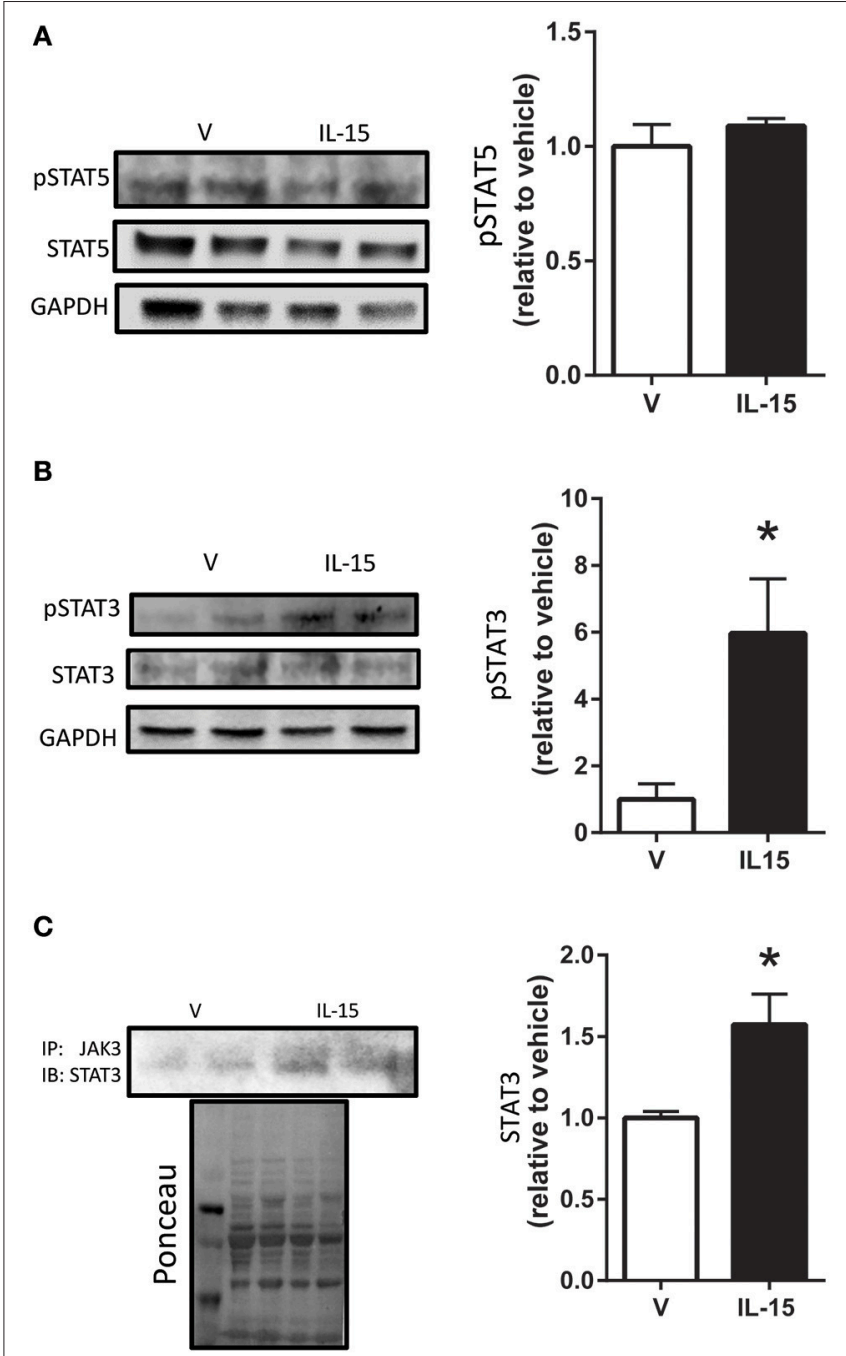

FIGURE 3 | Effects of IL-15 on STAT activation. Western blotting of (A) phospho-STAT5 and total STAT5; (B) phospho-STAT3 at Tyr705 and total STAT3; (C) immunoprecipitation of Jak3 and subsequent western blotting of STAT3. Assessments were carried out on differentiated C2C12 myotubes following $24 \mathrm{~h}$ treatment with $100 \mathrm{ng} / \mathrm{ml}$ of IL-15. GAPDH was used as loading control for western blotting. Ponceau S was used as a loading control for the immunoprecipitation analysis. Vehicle cells were used as a control to calculate protein expression. Image $\mathrm{J}$ was used to quantify band density in western blotting experiments. All values are displayed as means $\pm \mathrm{SEM}, n=6$ per group, ${ }^{*} P<0.05$.

membrane relative to the total cell GLUT4 expression $(P<0.05$; Figures 6B,C).

\section{DISCUSSION}

Data from this study solidifies the notion that the myokine IL-15 is directly involved in mediating glucose metabolism in SKM. Here we have brought to light a comprehensive signaling mechanism for IL-15 action in SKM cells. Our data shows that IL-15 promotes translocation of GLUT4 to the 


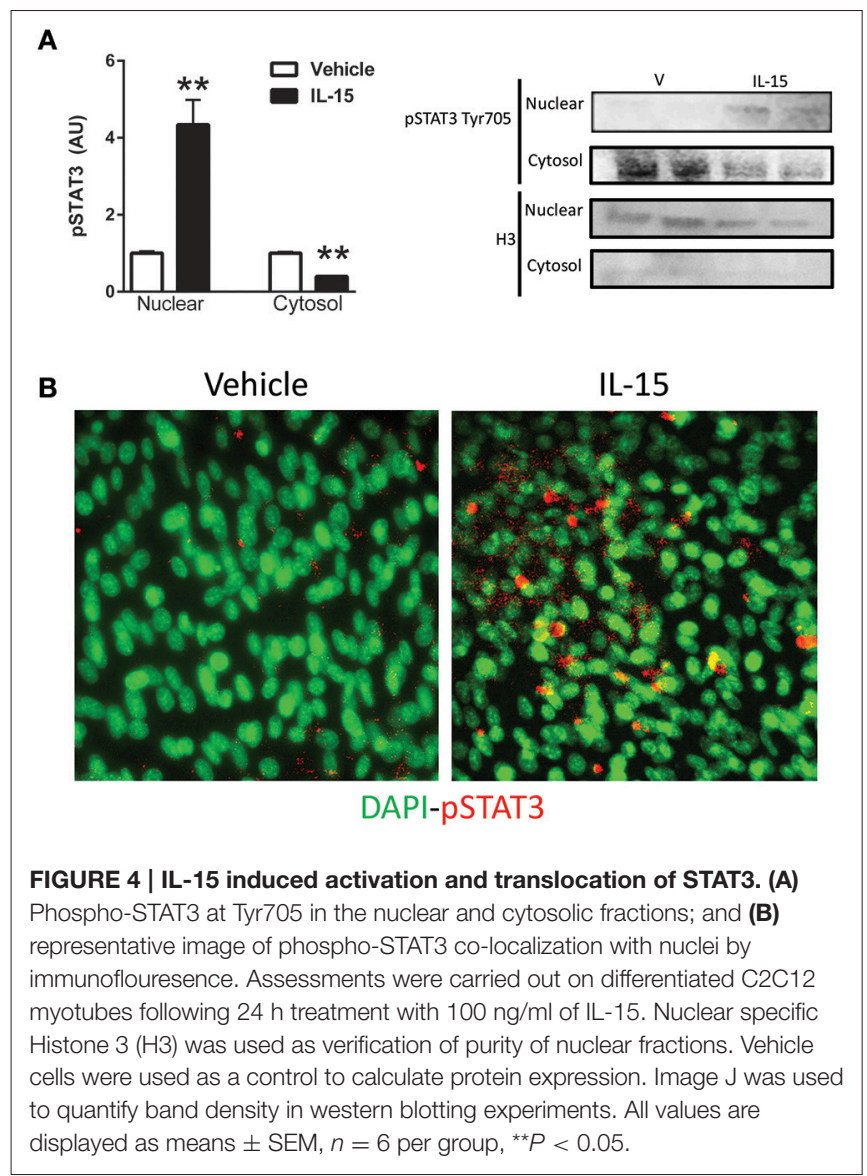

plasma membrane, accounting for the increase in glucose uptake. Additionally, our data indicates that IL-15 signals through the Jak3/STAT3 signaling pathway, without activation of other established downstream signals of IL-2R, in SKM cells. Moreover, we show for the first time that IL-15 promotes the translocation of phosphorylated STAT3 to the nucleus. The IL15 induced translocation of STAT3 might provide insight into the molecular mechanism behind the stimulatory effect of IL15 on overall cellular metabolism. Importantly, we uncovered a role for IL-15 induced STAT3 activation to induce HIF1 $\alpha$ expression, leading to an increase in GLUT4 translocation to the plasma membrane. Altogether, we provide evidence that IL-15 signaling in SKM is tightly regulated through coordination of the Jak3/STAT3 signaling pathway to promote GLUT4 translocation and consequently glucose uptake.

IL-15 has been extensively studied in tissues and cells other than SKM cells, such as NK lymphocytes (Lai et al., 2013; Mishra et al., 2014a). IL-15 signaling is complex, in that, it is ubiquitously expressed, but its secretion is proposed to be limited to SKM, and it is secreted with its receptor, IL-15R $\alpha$ (Budagian et al., 2006; Catoire et al., 2014). Complicating IL-15 signaling is its ability to trans-present in combination with IL-15R $\alpha$ to neighboring cells (Castillo and Schluns, 2012; Waldmann, 2015). Nevertheless, once released into circulation the IL-15/IL-15R $\alpha$ complex binds, or IL-15 acting alone, to the IL- $2 \mathrm{R} \beta / \gamma$ chains on its target tissue (Giri et al., 1995). It has repeatedly been shown

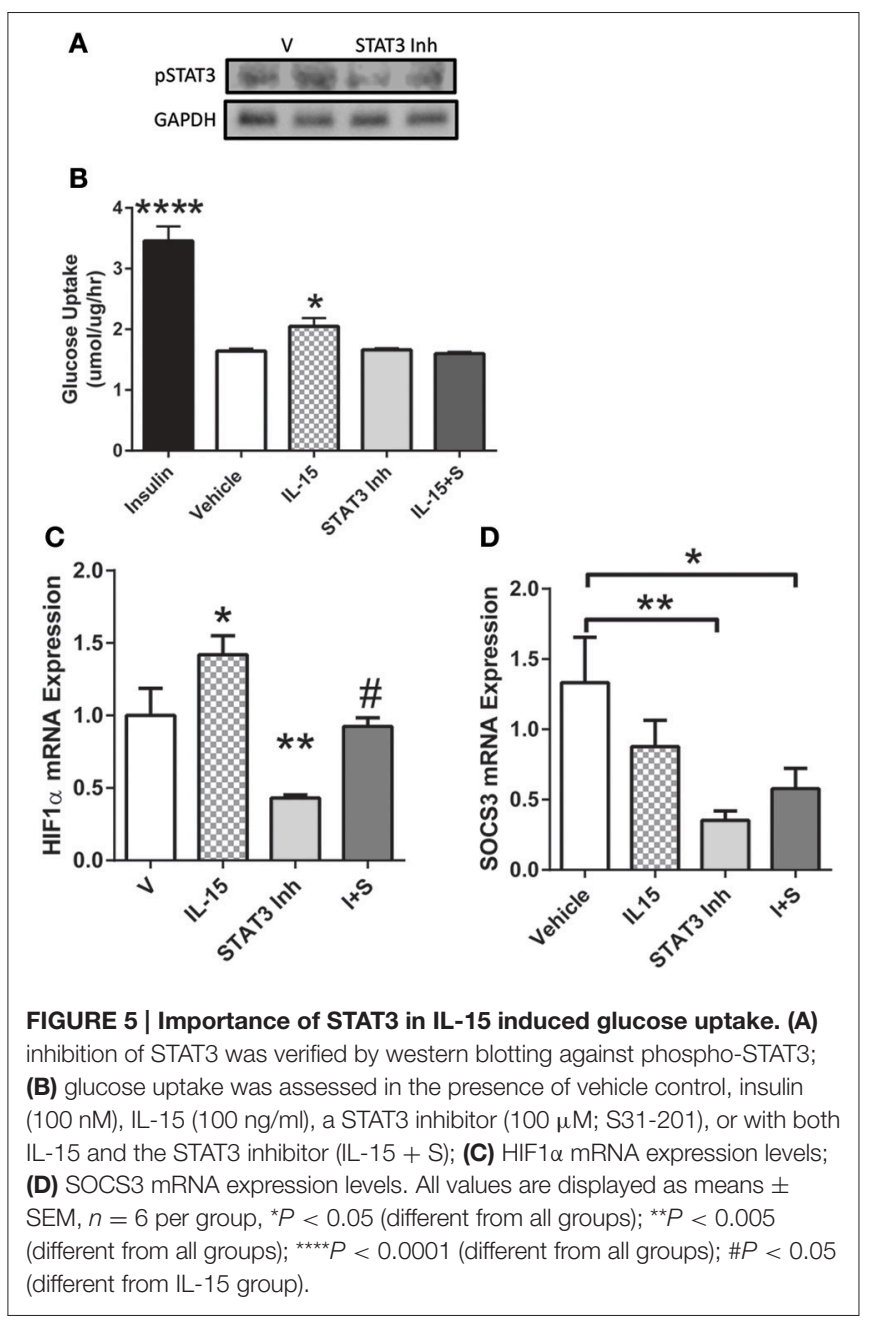

that IL-15 induces mitochondrial associated factors in various tissues, such as adipose and SKM (Almendro et al., 2006; Quinn et al., 2012, 2013; Barra et al., 2014; O'Connell and Pistilli, 2015). With this in mind, we examined the role of IL-15 signaling in mediating increases in substrate availability through modulation of transport proteins. We did not detect any alterations in total GLUT4 protein expression, which has been hypothesized to be controlled by IL-15 signaling (Busquets et al., 2006). However, in line with other studies, we measured increases in glucose uptake with IL-15 treatment in the SKM cells (Busquets et al., 2006). Further, we show that IL-15 acts to increase glucose uptake through induction of translocation of GLUT4 to the plasma membrane. GLUT4 translocation is a well-studied pathway and has been shown to occur via multiple regulators, such as AMPK (Thorell et al., 1999; Viollet et al., 2003). Additionally, it has been recognized that IL-15 acts upstream of proteins associated with mitochondrial activity, such as PGC1 $\alpha$, PPARs, and SIRT1 (Quinn et al., 2008, 2012; O'Connell and Pistilli, 2015). PGC1 $\alpha$ and PPAR signaling are downstream targets of the important energy sensing enzyme, AMPK, and us and others, have shown a relationship between AMPK and IL-15 in SKM. We previously showed that treatment with IL-15 reduced 


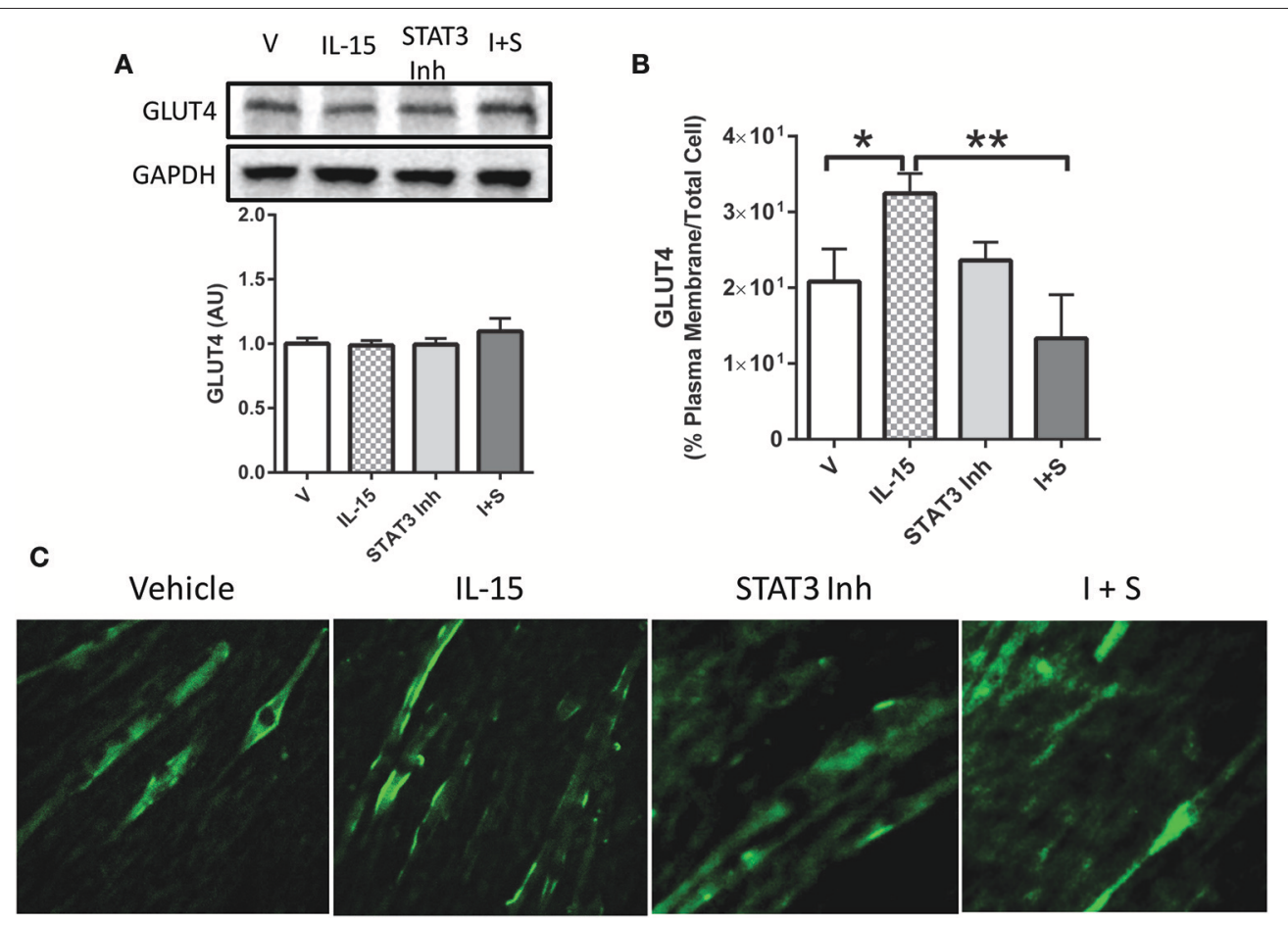

FIGURE 6 | Importance of STAT3 in IL-15 mediated GLUT4 translocation. (A) GLUT4 expression; (B) percent of GLUT4 expression in the plasma membrane relative to the total GLUT4 within the cell; (C) immunofluorescence of GLUT4 in myotubes. Cells with treated with either vehicle control, IL-15 (100 ng/ml), a STAT3 inhibitor (100 $\mu \mathrm{M}$; S31-201), or with both IL-15 and the STAT3 inhibitor (IL-15 + S). All values are displayed as means \pm SEM, $n=6$ per group, ${ }^{\star} P<0.05$; ${ }^{* \star} P<0.005$.

phosphorylated AMPK content in SKM cells. However, in those experiments cells were serum starved, which yields independent effects on AMPK. In this regard, we maintained our cells, in the current studies, in growth media including serum, to rule out signaling pathways beyond IL-15 signaling. Nevertheless, we hypothesized that the beneficial metabolic effects of IL-15 may signal through activation of AMPK. However, upon IL15 treatment, phosphorylation of AMPK remained unchanged (Abbott et al., 2012; Wan et al., 2014; Crane et al., 2015).

Binding of the IL-15/IL15R $\alpha$ complex to IL-2R $\beta / \gamma$ activates numerous signaling pathways, such as the Akt and Jak/STAT pathways, among others (Stone et al., 2011; Zhao and Huang, 2012; Lai et al., 2013). We speculated that IL-15 signals through the Akt signaling pathway, based on previously published work and our glucose kinetics data (Zhao and Huang, 2012). Interestingly, we were unable to measure increases in Akt activation by examination of its phosphorylation state and by assessing its downstream substrates, TBC1D1 and TBC1D4. The results of our investigation of Akt are in contradiction to other studies that point to a link between IL-15 and Akt signaling in SKM (Zhao and Huang, 2012; Lai et al., 2013). The study conducted by Zhao and Huang showed that IL-15 treatment, in combination with Akt overexpression, acted to regulate Tcell-SKM interaction following denervation (Zhao and Huang, 2012). However, in our study we examined the ability of IL-15 treatment to modulate the activation state of Akt in C2C12 SKM cells.
Ruling out AMPK and Akt activation, we next examined the Jak/STAT signaling pathway, which is widely accepted as a downstream modulator of IL-15/IL-2R signaling (Budagian et al., 2006; Lai et al., 2013; Waldmann, 2015; Ye, 2015). Not surprisingly, Jak3 phosphorylation was increased, as Jak3 has repeatedly been shown to be activated by IL-15/IL-2R signaling in numerous cells (Lai et al., 2013). On the other hand, some have shown that Jak1 is downstream of IL-15 signaling, but here, we failed to provide a link between IL-15 and Jak1 signaling, which is line with other reports (Kirken et al., 1995; Lai et al., 2013). Further, IL-15 failed to induce STAT5 phosphorylation, which is not in agreement with previous studies, showing STAT5 as downstream of the IL-2R and/or IL15 signaling (Lin and Leonard, 2000; Crane et al., 2015). Of note, little is known about the signaling molecules downstream of the IL-15 receptor, IL-2R, in SKM cells, which may account for the discrepancies between our data an others published in alternate cell types. Nevertheless, our data indicates that IL-15 signaling induces activation of STAT3, which is in line with other studies in endothelial cells (Stone et al., 2011). Importantly, we measured increased Jak3-STAT3 interaction, presumably to induce STAT3 phosphorylation at Tyr705, in the IL-15 treated SKM cells. It has been previously determined that upon phosphorylation and activation, from an upstream Jak, STAT3 translocates to the nucleus to bind to DNA, inducing its transcriptional activity (Yahata et al., 2003; Frias and Montessuit, 2013). In line with these aforementioned reports, we measured 
increased phosphorylated STAT3 content in the nuclear fraction of the SKM cells with IL-15 induction. Thus, translocation of STAT3, following phosphorylation at Tyr705, to the nucleus is an indicator of its transcriptional activity and is the potential route by which IL-15 promotes expression of genes associated with metabolic activity (Bild et al., 2002; Yahata et al., 2003). Since STAT3 activation has been associated with increases in glucose metabolism, our data provides a link for IL-15 induced metabolic function through Jak3/STAT3 signaling (Cui et al., 2004; Frias and Montessuit, 2013; Richard and Stephens, 2014). On the other hand, it has been suggested that STAT3-SOCS3 signaling stimulates insulin resistance in high fat diet induced obesity (Wunderlich et al., 2013). However, we can rule out SOCS3 as a player in the IL-15 signaling pathway, in regards to glucose metabolism, as its expression levels remained unchanged in the presence of IL-15. Adding to the controversy, when STAT3 was knocked out of skeletal muscle, in mice, there were no effects on whole body insulin tolerance, glucose uptake, fat oxidation, physical activity, or body weight (White et al., 2015). Nevertheless, here we can conclude, based on our data, that STAT3 activation, via IL-15, is indeed involved in mediating glucose uptake in SKM myotubes. Here we show that HIF1 $\alpha$, an established downstream target of STAT3, and mediator of glucose uptake and GLUT4 translocation, may be the link between IL-15Jak3/STAT3 and glucose uptake (Demaria et al., 2010; Sakagami et al., 2014).

Taken together, it is clear that downstream effects of the IL-15 signaling pathway, required to carry out its beneficial effects on metabolism, are complex and convoluted. Data presented here

\section{REFERENCES}

Abbott, M., and Turcotte, L. (2014). AMPK- $\alpha 2$ is involved in exercise training-induced adaptations in insulin-stimulated metabolism in skeletal muscle following high-fat diet. J. Appl. Physiol. (1985), 117, 869-879. doi: 10.1152/japplphysiol.01380.2013

Abbott, M. J., Constantinescu, S., and Turcotte, L. P. (2012). AMP-activated protein kinase $\alpha 2$ is an essential signal in the regulation of insulin-stimulated fatty acid uptake in control-fed and high-fat-fed mice. Exp. Physiol. 97, 603-617. doi: 10.1113/expphysiol.2012.064402

Abbott, M. J., Edelman, A. M., and Turcotte, L. P. (2009). CaMKK is an upstream signal of AMP-activated protein kinase in regulation of substrate metabolism in contracting skeletal muscle. Am. J. Physiol. Regul. Integr. Comp. Physiol. 297, R1724-R1732. doi: 10.1152/ajpregu.00179.2009

Ahmadian, M., Abbott, M. J., Tang, T., Hudak, C. S. S., Kim, Y., Bruss, M., et al. (2011). Desnutrin/ATGL is regulated by AMPK and is required for a brown adipose phenotype. Cell Metab. 13, 739-748. doi: 10.1016/j.cmet.2011.05.002

Almendro, V., Busquets, S., Ametller, E., Carbó, N., Figueras, M., Fuster, G., et al. (2006). Effects of interleukin-15 on lipid oxidation: disposal of an oral $\left[{ }^{14} \mathrm{C}\right]$-triolein load. Biochim. Biophys. Acta 1761, 37-42. doi: 10.1016/j.bbalip.2005.12.006

Almendro, V., Fuster, G., Busquets, S., Ametller, E., Figueras, M., Argilés, J. M., et al. (2008). Effects of IL-15 on rat brown adipose tissue: uncoupling proteins and PPARs. Obesity 16, 285-289. doi: 10.1038/oby.2007.47

Alvarez, B., Carbó, N., López-Soriano, J., Drivdahl, R. H., Busquets, S., López-Soriano, F. J., et al. (2002). Effects of interleukin-15 (IL-15) on adipose tissue mass in rodent obesity models: evidence for direct IL-15 action on adipose tissue. Biochim. Biophys. Acta 1570, 33-37. doi: 10.1016/S0304-4165(02)00148-4 provide evidence for direct regulation of IL-15 on glucose uptake in SKM cells. Additionally, we solidify a signaling mechanism for IL-15 action, through activation of Jak 3 to ultimately activate and promote STAT3 translocation to the nucleus. We propose a novel signaling mechanism for IL-15 mediated increases in glucose uptake and GLUT4 translocation via nuclear translocation of STAT3. Further studies are essential to firmly establish the unknown factors involved in the IL-15-Jak3/STAT3 signaling axis for the potential treatment and/or prevention of metabolic disorders, such as insulin resistance or diabetes.

\section{AUTHOR CONTRIBUTIONS}

JK, ST, and MA performed experiments and data collection. JK and ST edited and approved manuscript. MA devised experiments, performed statistical assessments, and wrote manuscript.

\section{FUNDING}

This work was supported by Chapman University, the American College of Sports Medicine Foundation, and the American Heart Association Grant No. 16SDG30680003 (MA).

\section{ACKNOWLEDGMENTS}

The authors would like to thank Allison Kawata, Ty Nakamoto, Kendra Rickart, and Brady Slater for their valuable technical expertise.

Argilés, J. M., López-Soriano, F. J., and Busquets, S. (2009). Therapeutic potential of interleukin-15: a myokine involved in muscle wasting and adiposity. Drug Discov. Today 14, 208-213. doi: 10.1016/j.drudis.2008.10.010

Barra, N. G., Palanivel, R., Denou, E., Chew, M. V., Gillgrass, A., Walker, T. D., et al. (2014). Interleukin-15 modulates adipose tissue by altering mitochondrial mass and activity. PLoS ONE 9:e114799. doi: 10.1371/journal.pone.0114799

Bild, A. H., Turkson, J., and Jove, R. (2002). Cytoplasmic transport of Stat3 by receptor-mediated endocytosis. EMBO J. 21, 3255-3263. doi: 10.1093/emboj/cdf351

Bogachus, L. D., and Turcotte, L. P. (2010). Genetic downregulation of AMPK- $\alpha$ isoforms uncovers the mechanism by which metformin decreases FA uptake and oxidation in skeletal muscle cells. Am. J. Physiol. Cell Physiol. 299, C1549-C1561. doi: 10.1152/ajpcell.00279.2010

Budagian, V., Bulanova, E., Paus, R., and Bulfone-Paus, S. (2006). IL-15/IL-15 receptor biology: a guided tour through an expanding universe. Cytokine Growth Factor Rev. 17, 259-280. doi: 10.1016/j.cytogfr.2006.05.001

Busquets, S., Figueras, M., Almendro, V., López-Soriano, F. J., and Argilés, J. M. (2006). Interleukin-15 increases glucose uptake in skeletal muscle. An antidiabetogenic effect of the cytokine. Biochim. Biophys. Acta 1760, 1613-1617. doi: 10.1016/j.bbagen.2006.09.001

Castillo, E. F., and Schluns, K. S. (2012). Regulating the immune system via IL-15 transpresentation. Cytokine 59, 479-490. doi: 10.1016/j.cyto.2012.06.017

Catoire, M., Mensink, M., Kalkhoven, E., Schrauwen, P., and Kersten, S. (2014). Identification of human exercise-induced myokines using secretome analysis. Physiol. Genomics 46, 256-267. doi: 10.1152/physiolgenomics.00174.2013

Crane, J. D., MacNeil, L. G., Lally, J. S., Ford, R. J., Bujak, A. L., Brar, I. K., et al. (2015). Exercise-stimulated interleukin-15 is controlled by AMPK and regulates skin metabolism and aging. Aging Cell 14, 625-634. doi: $10.1111 /$ acel.12341 
Cui, Y., Huang, L., Elefteriou, F., Yang, G., Shelton, J. M., Giles, J. E., et al. (2004). Essential role of STAT3 in body weight and glucose homeostasis. Mol. Cell. Biol. 24, 258-269. doi: 10.1128/MCB.24.1.258-269.2004

Demaria, M., Giorgi, C., Lebiedzinska, M., Esposito, G., D’Angeli, L., Bartoli, A., et al. (2010). A STAT3-mediated metabolic switch is involved in tumor transformation and STAT3 addiction. Aging (Albany N.Y.) 2, 823-842. doi: 10.18632/aging.100232

Eckardt, K., Görgens, S., Raschke, S., and Eckel, J. (2014). Myokines in insulin resistance and type 2 diabetes. Diabetologia 57, 1087-1099. doi: $10.1007 / \mathrm{s} 00125-014-3224-\mathrm{x}$

Egan, B., and Zierath, J. R. (2013). Exercise metabolism and the molecular regulation of skeletal muscle adaptation. Cell Metab. 17, 162-184. doi: 10.1016/j.cmet.2012.12.012

Febbraio, M. A. (2014). Role of interleukins in obesity: implications for metabolic disease. Trends Endocrinol. Metab. 25, 312-319. doi: 10.1016/j.tem.2014.02.004

Félix-Redondo, F. J., Grau, M., Baena-Díez, J. M., Dégano, I. R., de León, A. C., Guembe, M. J., et al. (2013). Prevalence of obesity and associated cardiovascular risk: the DARIOS study. BMC Public Health 13:542. doi: 10.1186/1471-2458-13-542

Fisher ffolliott, M., Kleiner, S., Douris, N., Fox, E. C., Mepani, R. J., Verdeguer, F., et al. (2012). FGF21 regulates PGC- $1 \alpha$ and browning of white adipose tissues in adaptive thermogenesis. Genes Dev. 26, 271-281. doi: 10.1101/gad.177857.111

Frias, M. A., and Montessuit, C. (2013). JAK-STAT signaling and myocardial glucose metabolism. JAKSTAT 2:e26458. doi: 10.4161/jkst.26458

Giri, J. G., Kumaki, S., Ahdieh, M., Friend, D. J., Loomis, A., Shanebeck, K., et al. (1995). Identification and cloning of a novel IL-15 binding protein that is structurally related to the alpha chain of the IL-2 receptor. EMBO J. 14, 3654-3663.

Gray, S. R., and Kamolrat, T. (2011). The effect of exercise induced cytokines on insulin stimulated glucose transport in C2C12 cells. Cytokine 55, 221-228. doi: $10.1016 /$ j.cyto.2011.04.019

Hampton, T. (2012). "Browning" of white fat may help in the ongoing fight against obesity. JAMA 308:1080. doi: 10.1001/2012.jama.11403

Indrakusuma, I., Sell, H., and Eckel, J. (2015). Novel mediators of adipose tissue and muscle crosstalk. Curr. Obes. Rep. 4, 411-417. doi: $10.1007 / \mathrm{s} 13679-015-0174-7$

Ivy, J. L., Sherman, W. M., Cutler, C. L., and Katz, A. L. (1986). Exercise and diet reduce muscle insulin resistance in obese Zucker rat. Am. J. Physiol. Endocrinol. Metab. 251, E299-E305.

Kanwal, A., Singh, S. P., Grover, P., and Banerjee, S. K. (2012). Development of a cell-based nonradioactive glucose uptake assay system for SGLT1 and SGLT2. Anal. Biochem. 429, 70-75. doi: 10.1016/j.ab.2012.07.003

Kelly, K. R., Abbott, M. J., and Turcotte, L. P. (2010). Short-term AMP-regulated protein kinase activation enhances insulin-sensitive fatty acid uptake and increases the effects of insulin on fatty acid oxidation in L6 muscle cells. Exp. Biol. Med. (Maywood N.J.) 235, 514-521. doi: 10.1258/ebm.2009.009228

Kirken, R. A., Rui, H., Malabarba, M. G., Howard, O. M., Kawamura, M., O'Shea, J. J., et al. (1995). Activation of JAK3, but not JAK1, is critical for IL-2-induced proliferation and STAT5 recruitment by a $\mathrm{COOH}$-terminal region of the IL-2 receptor $\beta$-chain. Cytokine 7, 689-700. doi: 10.1006/cyto.19 95.0081

Kraegen, E. W., Storlien, L. H., Jenkins, A. B., and James, D. E. (1989). Chronic exercise compensates for insulin resistance induced by a high-fat diet in rats. Am. J. Physiol. Endocrinol. Metab. 256, E242-E249.

Lai, Y. G., Hou, M. S., Lo, A., Huang, S. T., Huang, Y. W., Yang-Yen, H. F., et al. (2013). IL-15 modulates the balance between Bcl-2 and Bim via a Jak3/1-PI3KAkt-ERK pathway to promote CD8 $\alpha \alpha^{+}$intestinal intraepithelial lymphocyte survival. Eur. J. Immunol. 43, 2305-2316. doi: 10.1002/eji.201243026

Leira, F., Louzao, M. C., Vieites, J. M., Botana, L. M., and Vieytes, M. R. (2002). Fluorescent microplate cell assay to measure uptake and metabolism of glucose in normal human lung fibroblasts. Toxicol. Vitr. 16, 267-273. doi: $10.1016 / \mathrm{S} 0887-2333(02) 00002-4$

Lin, J.-X., and Leonard, W. J. (2000). The role of Stat5a and Stat5b in signaling by IL-2 family cytokines. Oncogene 19, 2566-2576. doi: 10.1038/sj.onc. 1203523

Lutz, C. T., and Quinn, L. S. (2012). Sarcopenia, obesity, and natural killer cell immune senescence in aging: altered cytokine levels as a common mechanism. Aging (Albany N.Y.) 4, 535-546. doi: 10.18632/aging.100482
Mishra, A., Liu, S., Sams, G. H., Curphey, D. P., Santhanam, R., Rush, L. J., et al. (2014a). Aberrant overexpression of IL-15 initiates large granular lymphocyte leukemia through chromosomal instability and DNA hypermethylation. Cancer Cell 22, 645-655. doi: 10.1016/j.ccr.2012.09.009

Mishra, A., Sullivan, L., and Caligiuri, M. A. (2014b). Molecular pathways: interleukin-15 signaling in health and in cancer. Clin. Cancer Res. 20, 2044-2050. doi: 10.1158/1078-0432.CCR-12-3603

O'Connell, G. C., and Pistilli, E. E. (2015). Interleukin-15 directly stimulates prooxidative gene expression in skeletal muscle in-vitro via a mechanism that requires interleukin-15 receptor alpha. Biochem. Biophys. Res. Commun. 458, 614-619. doi: 10.1016/j.bbrc.2015.02.015

Ogden, C. L., Carroll, M. D., Kit, B. K., and Flegal, K. M. (2014). Prevalence of childhood and adult obesity in the united states, 2011-2012. JAMA 311, 806-814. doi: 10.1001/jama.2014.732

Pedersen, B. K. (2013). Muscle as a secretory organ. Compr. Physiol. 3, 1337-1362. doi: $10.1002 /$ cphy.c120033

Pedersen, B. K., and Febbraio, M. A. (2012). Muscles, exercise and obesity: skeletal muscle as a secretory organ. Nat. Rev. Endocrinol. 8, 457-465. doi: 10.1038/nrendo.2012.49

Pierce, J. R., Maples, J. M., and Hickner, R. C. (2015). IL-15 concentrations in skeletal muscle and subcutaneous adipose tissue in lean and obese humans: local effects of IL-15 on adipose tissue lipolysis. Am. J. Physiol. Endocrinol. Metab. 308, E1131-E1139. doi: 10.1152/ajpendo.00575.2014

Quinn, L. S., and Anderson, B. G. (2011). Interleukin-15, IL-15 receptor-alpha, and obesity: concordance of laboratory and human genetic studies. J. Obes. 2011:8. doi: $10.1155 / 2011 / 456347$

Quinn, L. S., Anderson, B. G., Conner, J. D., and Wolden-Hanson, T. (2012). IL-15 overexpression promotes endurance, oxidative energy metabolism, and muscle PPAR $\delta$, SIRT1, PGC- $1 \alpha$, and PGC- $1 \beta$ expression in male mice. Endocrinology 154, 232-245. doi: 10.1210/en.2012-1773

Quinn, L. S., Anderson, B. G., Conner, J. D., Wolden-Hanson, T., and Marcell, T. J. (2013). IL-15 is required for postexercise induction of the pro-oxidative mediators PPAR $\delta$ and SIRT1 in male mice. Endocrinology 155, 143-155. doi: 10.1210/en.2013-1645

Quinn, L. S., Anderson, B. G., Strait-Bodey, L., Stroud, A. M., and Argilés, J. M. (2008). Oversecretion of interleukin-15 from skeletal muscle reduces adiposity. Am. J. Physiol. Endocrinol. Metab. 296, E191-E202. doi: 10.1152/ajpendo.90506.2008

Raschke, S., and Eckel, J. (2013). Adipo-myokines: two sides of the same coin-mediators of inflammation and exercise. Mediators Inflamm. 2013:16. doi: $10.1155 / 2013 / 320724$

Richard, A. J., and Stephens, J. M. (2014). The role of JAK-STAT signaling in adipose tissue function. Biochim. Biophys. Acta 1842, 431-439. doi: 10.1016/j.bbadis.2013.05.030

Rinnov, A., Yfanti, C., Nielsen, S., Åkerström, T. A., Peijs, L., Zankari, A., et al. (2014). Endurance training enhances skeletal muscle interleukin-15 in human male subjects. Endocrine 45, 271-278. doi: 10.1007/s12020-013-9969-z

Sakagami, H., Makino, Y., Mizumoto, K., Isoe, T., Takeda, Y., Watanabe, J., et al. (2014). Loss of HIF-1 $\alpha$ impairs GLUT4 translocation and glucose uptake by the skeletal muscle cells. Am. J. Physiol. Endocrinol. Metab. 306, E1065-E1076. doi: 10.1152/ajpendo.00597.2012

Schindelin, J., Arganda-Carreras, I., Frise, E., Kaynig, V., Longair, M., Pietzsch, T., et al. (2012). Fiji: an open-source platform for biological-image analysis. Nat. Meth. 9, 676-682. doi: 10.1038/nmeth.2019

Schneider, C. A., Rasband, W. S., and Eliceiri, K. W. (2012). NIH Image to ImageJ: 25 years of image analysis. Nat. Methods 9, 671-675. doi: 10.1038/nmeth.2089

Siddiquee, K., Zhang, S., Guida, W. C., Blaskovich, M. A., Greedy, B., Lawrence, H. R., et al. (2007). Selective chemical probe inhibitor of Stat3, identified through structure-based virtual screening, induces antitumor activity. Proc. Natl. Acad. Sci. U.S.A. 104, 7391-7396. doi: 10.1073/pnas.0609757104

Stone, K. P., Kastin, A. J., and Pan, W. (2011). NFкB is an unexpected major mediator of interleukin-15 signaling in cerebral endothelia. Cell. Physiol. Biochem. 28, 115-124. doi: 10.1159/000331720

Sun, H., and Liu, D. (2015). Hydrodynamic delivery of interleukin 15 gene promotes resistance to high fat diet-induced obesity, fatty liver and improves glucose homeostasis. Gene Ther. 22, 341-347. doi: 10.1038/gt.20 14.114 
Tammineni, P., Anugula, C., Mohammed, F., Anjaneyulu, M., Larner, A. C., and Sepuri, N. B. (2013). The import of the transcription factor STAT3 into mitochondria depends on GRIM-19, a component of the electron transport chain. J. Biol. Chem. 288, 4723-4732. doi: 10.1074/jbc.M112. 378984

Thorell, A., Hirshman, M. F., Nygren, J., Jorfeldt, L., Wojtaszewski, J. F. P., Dufresne, S. D., et al. (1999). Exercise and insulin cause GLUT-4 translocation in human skeletal muscle. Am. J. Physiol. Endocrinol. Metab. 277, E733-E741.

Thornton, S., Krolopp, J., and Abbott, M. J. (2016). IL-15 mediates mitochondrial activity through a PPAR $\delta$-dependent-PPAR $\alpha$-independent mechanism in skeletal muscle cell. PPAR Res. 2016:12. doi: 10.1155/2016/54 65804

Turcotte, L. P., and Abbott, M. J. (2012). Contraction-induced signaling: evidence of convergent cascades in the regulation of muscle fatty acid metabolism. Can. J. Physiol. Pharmacol. 90, 1419-1433. doi: 10.1139/y2012-124

Viollet, B., Andreelli, F., Jørgensen, S. B., Perrin, C., Geloen, A., Flamez, D., et al. (2003). The AMP-activated protein kinase $\alpha 2$ catalytic subunit controls whole-body insulin sensitivity. J. Clin. Invest. 111, 91-98. doi: 10.1172/ JCI16567

Waldmann, T. A. (2015). The shared and contrasting roles of IL2 and IL15 in the life and death of normal and neoplastic lymphocytes: implications for cancer therapy. Cancer Immunol. Res. 3, 219-227. doi: 10.1158/2326-6066.CIR-15-0009

Wan, Z., Root-Mccaig, J., Castellani, L., Kemp, B. E., Steinberg, G. R., and Wright, D. C. (2014). Evidence for the role of AMPK in regulating PGC-1 alpha expression and mitochondrial proteins in mouse epididymal adipose tissue. Obesity 22, 730-738. doi: 10.1002/oby.20605

White, A. T., LaBarge, S. A., McCurdy, C. E., and Schenk, S. (2015). Knockout of STAT3 in skeletal muscle does not prevent high-fat diet-induced insulin resistance. Mol. Metab. 4, 569-575. doi: 10.1016/j.molmet.2015.05.001
Winder, W. W., and Hardie, D. G. (1999). AMP-activated protein kinase, a metabolic master switch: possible roles in Type 2 diabetes. Am. J. Physiol. 277(1 Pt 1), E1-E10.

Wunderlich, C. M., Hövelmeyer, N., and Wunderlich, F. T. (2013). Mechanisms of chronic JAK-STAT3-SOCS3 signaling in obesity. JAKSTAT 2:e23878. doi: $10.4161 /$ jkst.23878

Yahata, Y., Shirakata, Y., Tokumaru, S., Yamasaki, K., Sayama, K., Hanakawa, Y., et al. (2003). Nuclear translocation of phosphorylated STAT3 is essential for vascular endothelial growth factor-induced human dermal microvascular endothelial cell migration and tube formation. J. Biol. Chem. 278, 40026-40031. doi: 10.1074/jbc.M301866200

Ye, J. (2015). Beneficial metabolic activities of inflammatory cytokine interleukin 15 in obesity and type 2 diabetes. Front. Med. 9, 139-145. doi: 10.1007/s11684-015-0377-z

Zhao, H., and Huang, H. (2012). Functional capability of IL-15-Akt signaling in the denervated muscle. Cytokine 60, 608-615. doi: 10.1016/j.cyto.2012.08.026

Zou, C., Wang, Y., and Shen, Z. (2005). 2-NBDG as a fluorescent indicator for direct glucose uptake measurement. J. Biochem. Biophys. Methods 64, 207-215. doi: 10.1016/j.jbbm.2005.08.001

Conflict of Interest Statement: The authors declare that the research was conducted in the absence of any commercial or financial relationships that could be construed as a potential conflict of interest.

Copyright (c) 2016 Krolopp, Thornton and Abbott. This is an open-access article distributed under the terms of the Creative Commons Attribution License (CC BY). The use, distribution or reproduction in other forums is permitted, provided the original author(s) or licensor are credited and that the original publication in this journal is cited, in accordance with accepted academic practice. No use, distribution or reproduction is permitted which does not comply with these terms. 